\title{
The effectiveness of lifestyle interventions to reduce side effects of androgen deprivation therapy for men with prostate cancer: a systematic review
}

\author{
Maud J. M. Geerkens ${ }^{1}$ (D) Nieck S. A. Pouwels ${ }^{1} \cdot$ Harry P. Beerlage $^{1}$
}

Accepted: 4 November 2019 / Published online: 12 December 2019

(c) The Author(s) 2019

\begin{abstract}
Purpose The aim of this review is to systematically review randomized controlled trials on lifestyle interventions on PCa patients undergoing androgen deprivation therapy.

Methods A literature search was conducted using the electronic databases Medline and PubMed. To be eligible, studies had to be randomized controlled trials (RCTs) that focused on side effects of ADT and lifestyle interventions to reduce side effects for men undergoing ADT with PCa. Lifestyle interventions were defined as interventions that included any dietary or behavioral components.

Results Twenty-nine trials were included. Most of them focused on exercise interventions, while some investigated the effect of dietary or behavioral interventions. The effect of different lifestyle influencing modalities aimed to improve on the adverse effects of ADT varied greatly.

Conclusions It is not possible to draw one conclusion on the effect of exercise-based interventions, but noted on several adverse effects of ADT improvement. Further studies are necessary to develop personalized lifestyle interventions in order to mitigate the adverse effects.
\end{abstract}

Keywords Males · ADT · Side effects · Prostate cancer · Lifestyle interventions

\section{Introduction}

Prostate cancer is one of the most common forms of cancer in men and the second cause of death [1]. Androgens and androgen receptor signaling play an important role in the normal growth and function of the prostate, but also in the development and maintenance of prostate cancer. The beneficial effect of castration in the treatment of prostate cancer has already been discovered in 1941, by Huggins [2]. LHRH agonists are the most chosen form of chemical castration [3]. LHRH agonists bring the serum testosterone to a level similar to castration by interfering in the pulsatile release of LHRH in the hypothalamus, thereby down-regulating the release of luteinizing hormone in the anterior pituitary

Maud J. M. Geerkens

maudgeerkens@hotmail.com

1 Urology Department, Amsterdam UMC locatie AMC Netherlands, Meibergdreef 9, 1105 AZ Amsterdam, The Netherlands gland. Androgen deprivation therapy (ADT) achieves a remission in 80-90\% in men with advanced prostate cancer and an average progression-free interval of 12-33 months [4].

Despite the fact that this form of therapy is very successful, it also known for its side effects and the impact of these side effects on the quality of life in addition to the psychological and physical effects influencing the quality of life in the long term. The most common life quality diminishing side effects are reduced libido, depression, fatigue, gynecomastia, hot flushes, obesity, hypertension, insulin resistance, and osteoporosis [5].

Non-pharmaceutical lifestyle interventions aimed to reduce side effects while aiming to leave control in the hands of the patient are very important. They may prevent medicalization and are thought to increase quality of life.

Previous reviews have shown that physical activity may alleviate side effects of ADT [6, 7]. These reviews mainly focused on the effects of different types of exercise, but did not include all types of lifestyle interventions. The aim of this research is to provide a comprehensive overview of 
recent physical and psychological lifestyle interventions to reduce different side effects of ADT and to investigate the impact of these interventions on quality of life.

\section{Methods}

\section{Data acquisition and search strategy}

A literature search was conducted using the electronic databases Medline and PubMed. The literature search included relevant publications until January 25, 2019. Predefined search terms were used to identify articles concerning interventions to reduce side effects of ADT used as a therapy for prostate cancer.

\section{Eligibility criteria}

To be eligible, the study population had to consist of patients diagnosed with local or advanced PCa in whom ADT was started. Studies had to be randomized controlled trials (RCTs) that focused on side effects of ADT and lifestyle interventions to reduce those side effects. For this review, we selected studies concerning the following psychological effects: quality of life (health-related quality of life and disease-specific quality of life), fatigue, reduced libido, and depression. We selected studies investigating the following physical side effects: gynecomastia, hot flushes, osteoporosis, obesity, or a decreased cardiovascular health. Lifestyle interventions were defined as interventions that included any dietary or behavioral components. Studies reporting on medical therapies to diminish side effects were excluded, as were studies in which the participants stopped their ADT and studies not related to humans.

\section{Screening of abstracts and full-text articles}

A search was performed for abstracts that may be used for inclusion. Abstract screening was done according to predefined inclusion and exclusion criteria. Full-text original articles were retrieved from the selected abstracts; only articles published in English or Dutch that were available for review were selected. Abstracts and original articles were independently assessed by two reviewers for eligibility (MG and NP). Disagreements were solved by consensus procedure in which an independent third author (HBLG) was conducted.

Subsequently, reference lists of all full-text articles were screened to identify additional relevant articles not found in the PubMed and Medline databases. The final number of included and excluded studies is illustrated in the Preferred Reporting Items for Systematic Reviews and Meta-analyses (Fig. 1).

\section{Study quality assessment tool}

The Cochrane Collaboration's tool was used to assess the risk of bias in the randomized controlled trial [8]. Two reviewers (MG and NP) assessed the overall quality. If no agreement could be reached, an independent person was involved (HPB).

\section{Data synthesis}

The following data were independently extracted from fulltext articles by two reviewers: study year, study design, study population, mean age, type of interventions, duration and frequency of interventions, relevant study outcomes, and methods to assess these outcomes.

\section{Results}

\section{Search results and analysis}

Our literature search identified 1961 of which 29 articles were included in this analysis. The studies were all RCTs investigating the effect of life style interventions to mitigate ADT-induced side effects. Twenty studies investigated exercise modalities, two studies investigated dietary advice, and four studies combined these methods. Three studies investigated behavioral components, existing of cognitive behavioral therapy, educational support programs, or self-education. Study characteristics are depicted in Table 1.

Table 2 shows an overview of RCTs testing mitigation strategies for ADT-induced side effects, categorized per side effect. Table 3 summarizes the quality scores of the RCTs. The quality differed between the studies. The most common source of methodological bias was a lack of proper blinding procedures. Almost one-third of the studies presented incomplete outcome data.

\section{Psychological side effects}

Quality of life Health-related quality of life (HRQOL) is perceived physical and mental health over time either by an individual or by a group. The influence of lifestyle interventions on the quality of life for patients on ADT was examined in 16 studies [9-15, 17, 18, 20, 23-27, 34, 36].

Ten different questionnaires were used to measure HRQOL. Some of the questionnaires are developed to examine health-related quality of life in general (STAI; SF-36; EORTC-QLQ- C30; QLQ-PR25; PSS; FACT-G; LFDI). Others are used to investigate prostatic disease-specific quality of life or aspects of quality of life (FACT-P; PORPUS; 
Fig. 1 Flow chart of the search and selection process

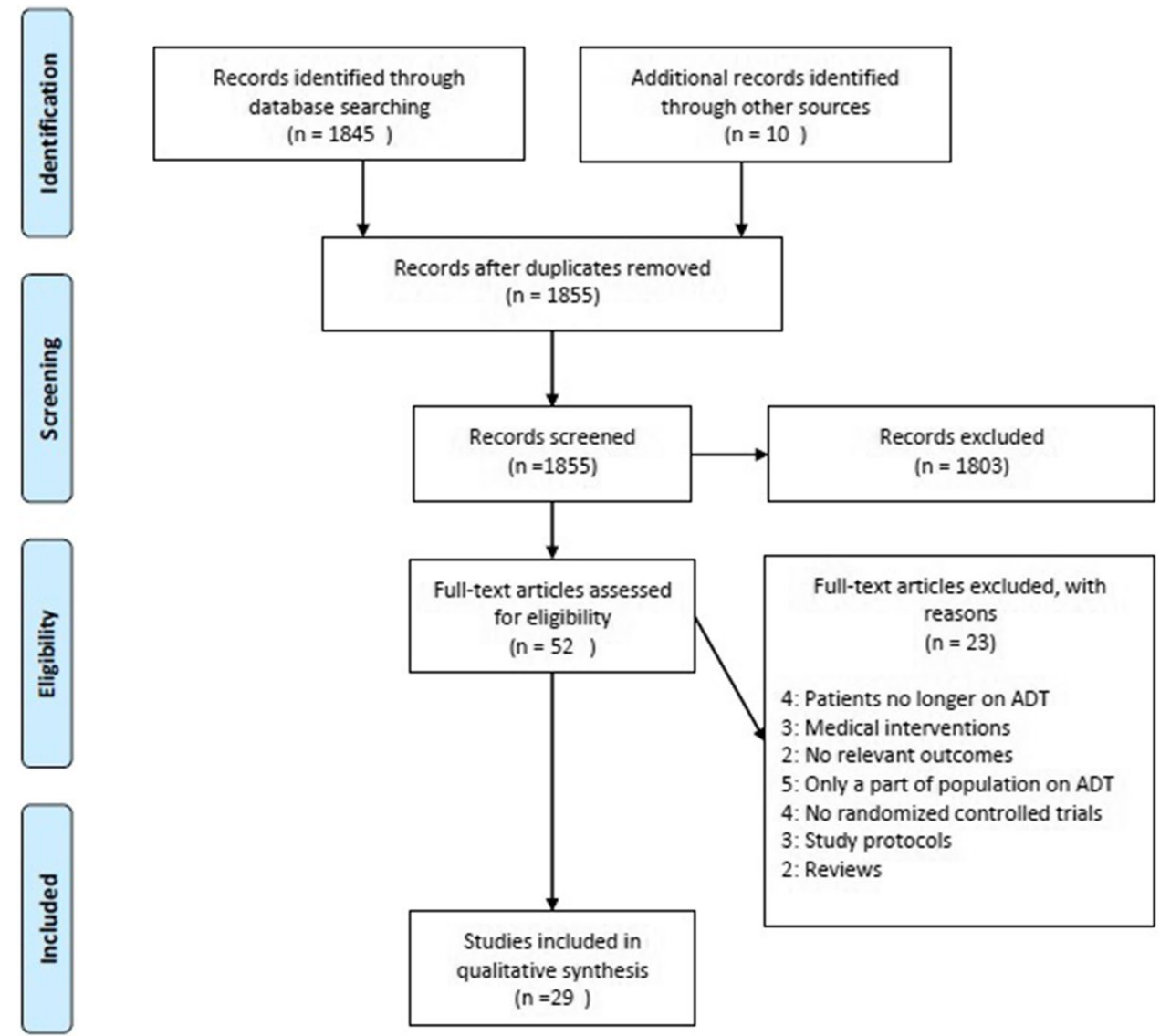

EPIC). Eleven studies investigated the effects of physical exercises, three combined physical exercises with dietary advice, and two investigated the effect of soy consumption. In four studies, a positive effect of exercise only was noted $[9,13,24,36]$. Two out of six studies investigating the effect of resistance training found a positive effect on the health-related quality of life and in one of these studies, an improved disease-specific quality of life had been found [36]. Aerobic and resistance training combined showed a positive effect in two out of four studies [13,24]. In these two studies, only specific domains were significantly improved. For details, see Table 2. Variable results were found when combining exercises with dietary advice. All three studies found positive effects on different aspects of quality of life [15, $20,26]$. Bourke et al. found an improvement in the prostatic disease-specific quality of life after 12 weeks of training, but this was only temporary since the effect disappeared after 24 weeks [20]. One study investigated the effect of cognitive behavioral therapy on quality of life, but failed to show an improvement. Two studies investigated the effect of soy consumption. Vitolins et al. found an improved HRQOL as well as an improved disease-specific quality of life after consumption of soy protein, while Sharma et al. failed to show any effect $[11,18]$.
Depression In the treatment of PCa, ADT use is associated with depression. Five studies focused on depression [10, 12, $24,27,36]$. Aerobic and/or resistance training failed to show a beneficial effect $[12,24,36]$. Applied cognitive behavioral therapy (CBT) for a period of four weeks did not show a beneficial effect [27]. A lifestyle activity program or an educational support program did not influence depression scores using CES-D [10].

Fatigue Fatigue is a phenomenon, which is difficult to measure or define. Fatigue is experienced by patients receiving ADT and is associated with decreased levels of testosterone and reduction of skeletal muscle mass may contribute to fatigue. Influence of lifestyle interventions was examined in fourteen studies: ten investigated the effects of physical exercises, three combined physical exercises with dietary advice, and one investigated the effect of soy consumption $[9,11-14,17,20,24-26,31,35,36]$. Soy consumption showed no effect [11].

Different exercise modalities yield conflicting results in relation to fatigue. Generally speaking, in half of the studies, a reduction in fatigue was noted. Combining exercises with dietary advice showed a beneficial effect in two studies [14, 20]. Another study failed to show improvement [26]. 


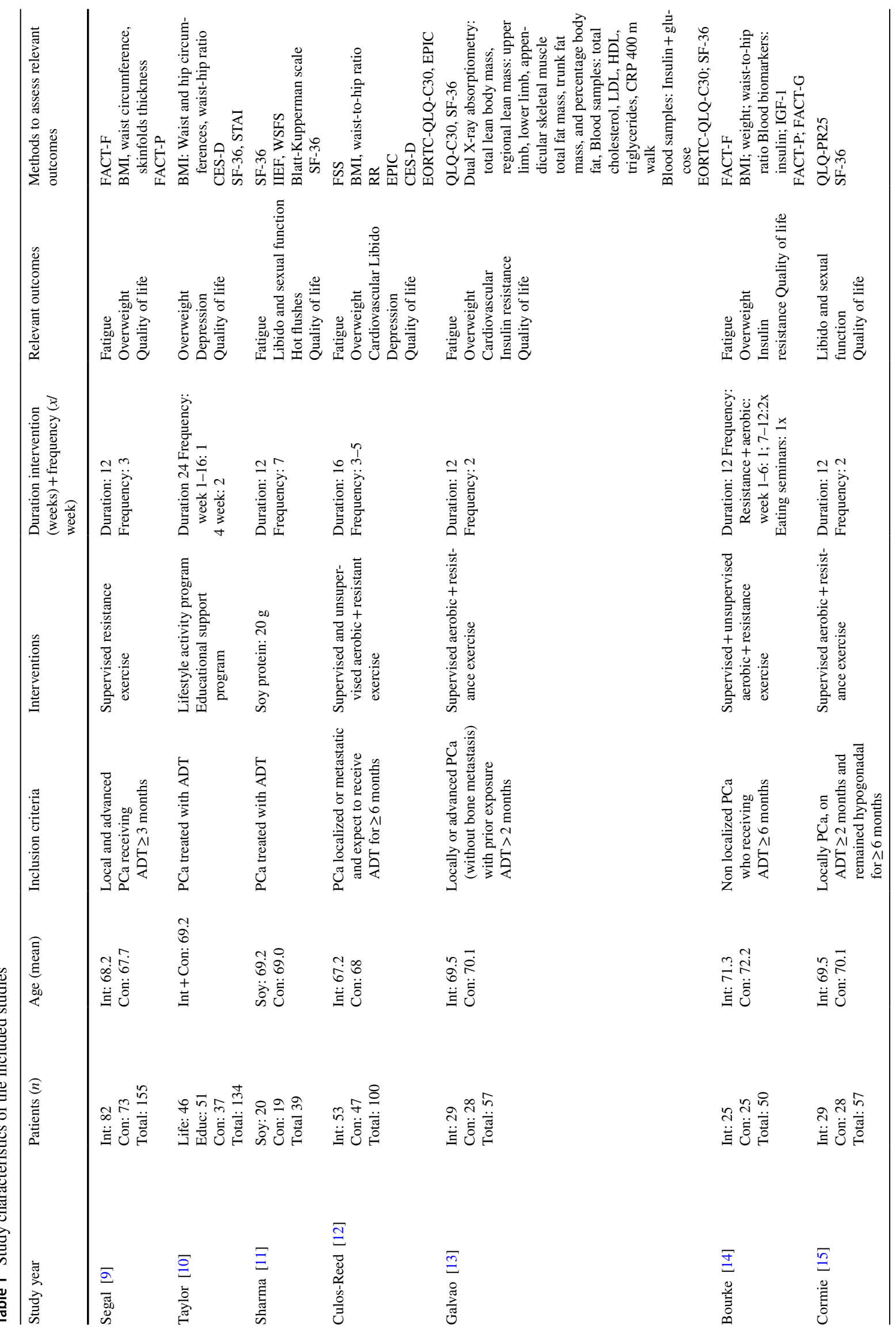




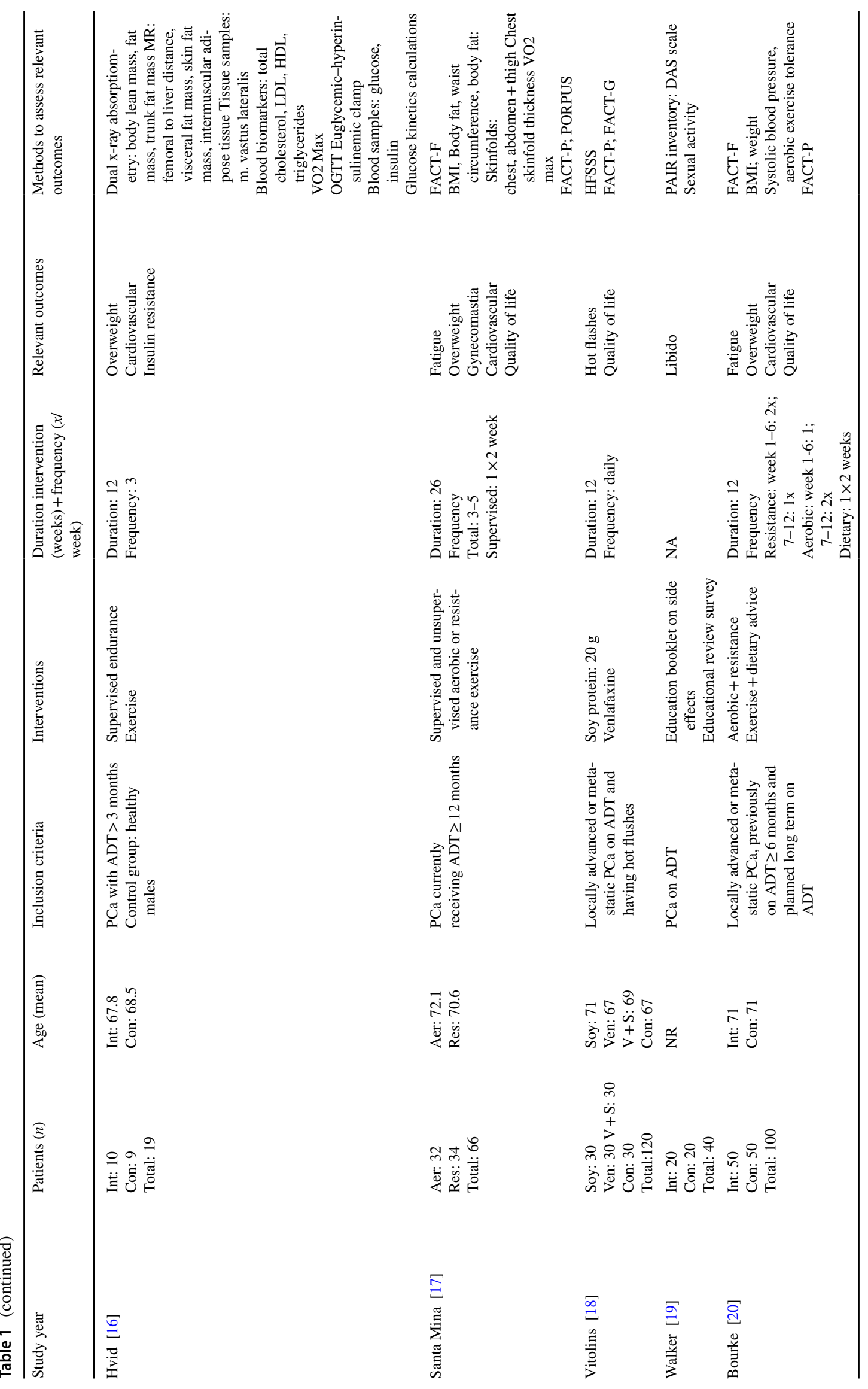


848

Quality of Life Research (2020) 29:843-865

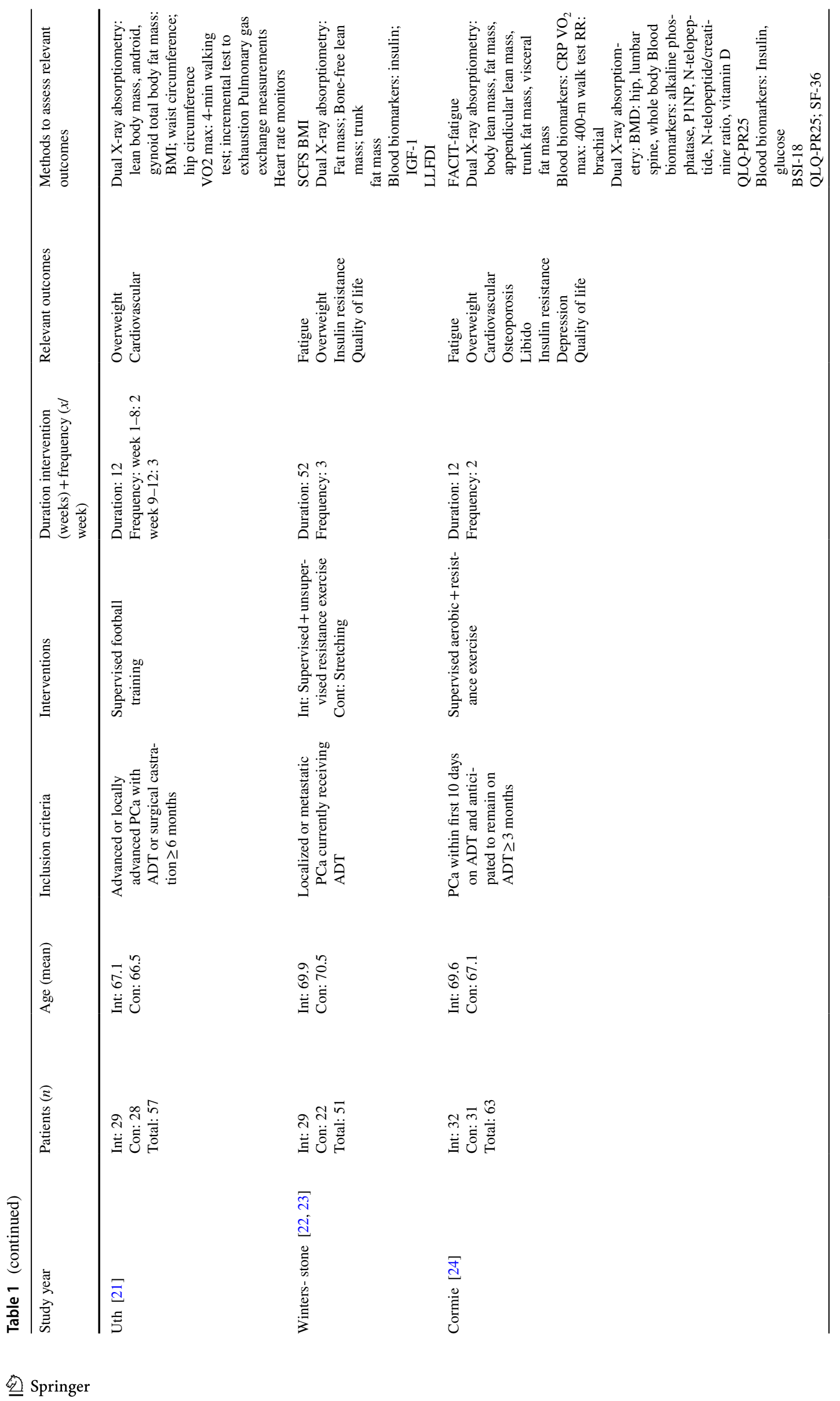




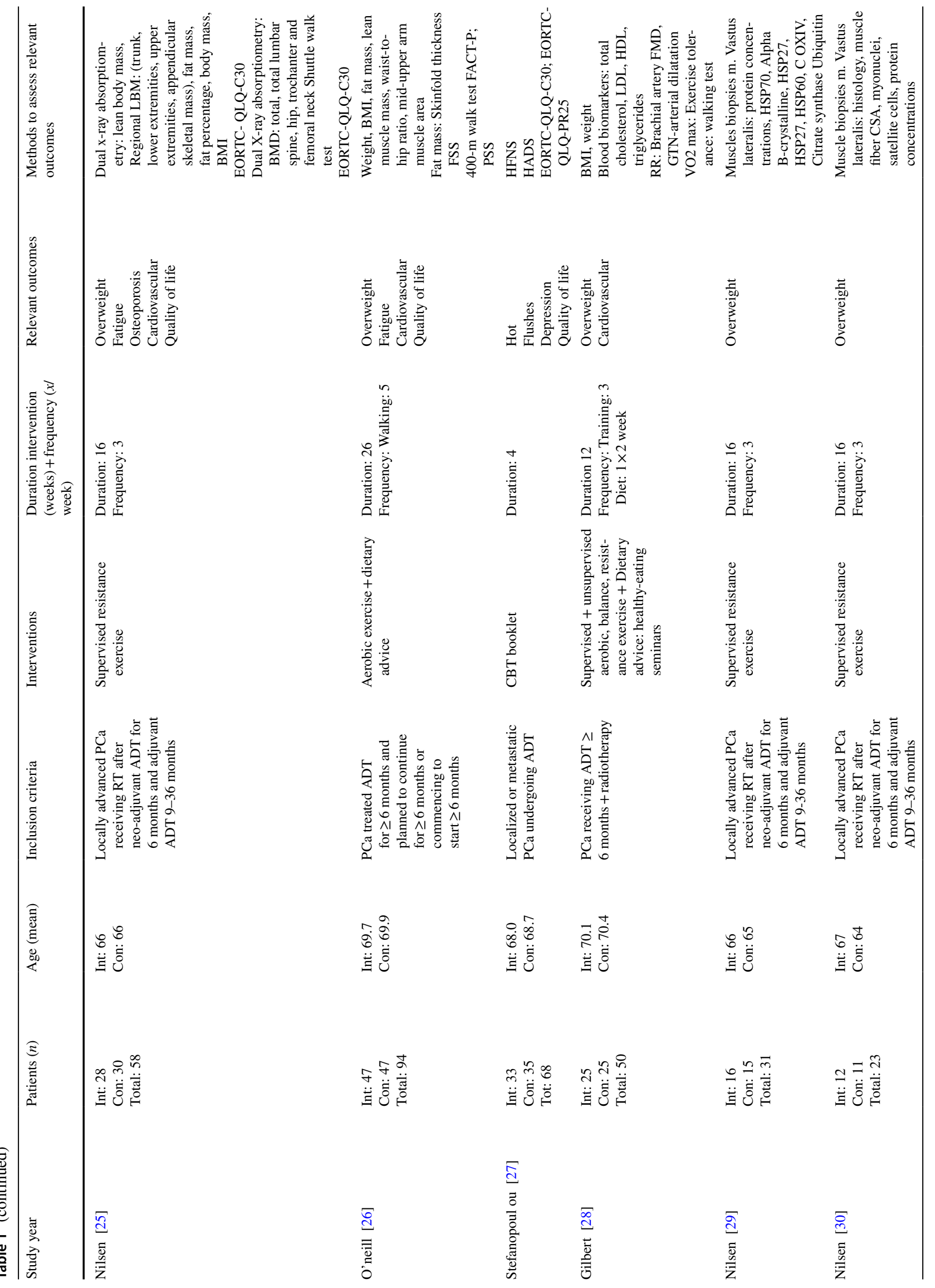




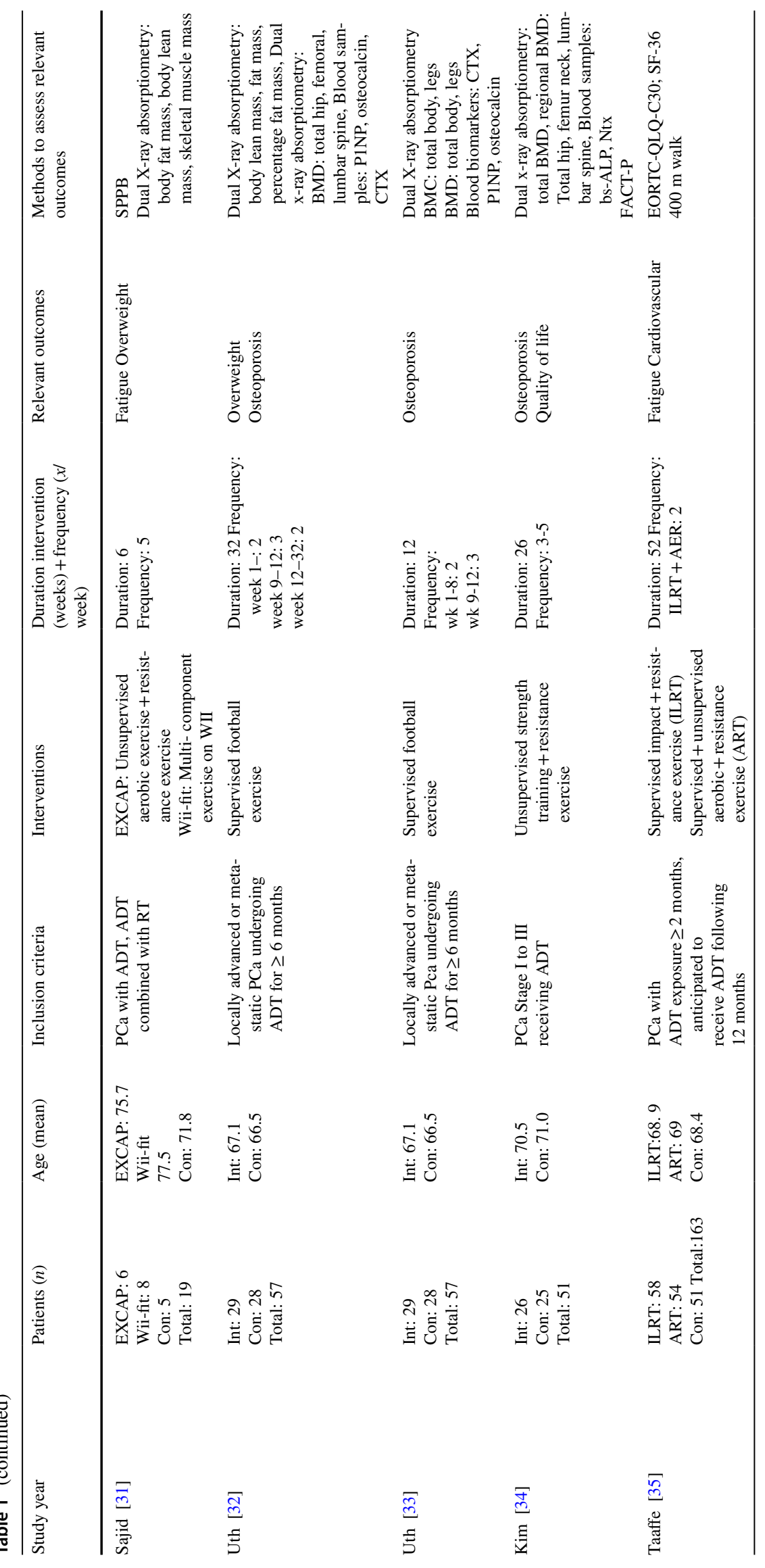




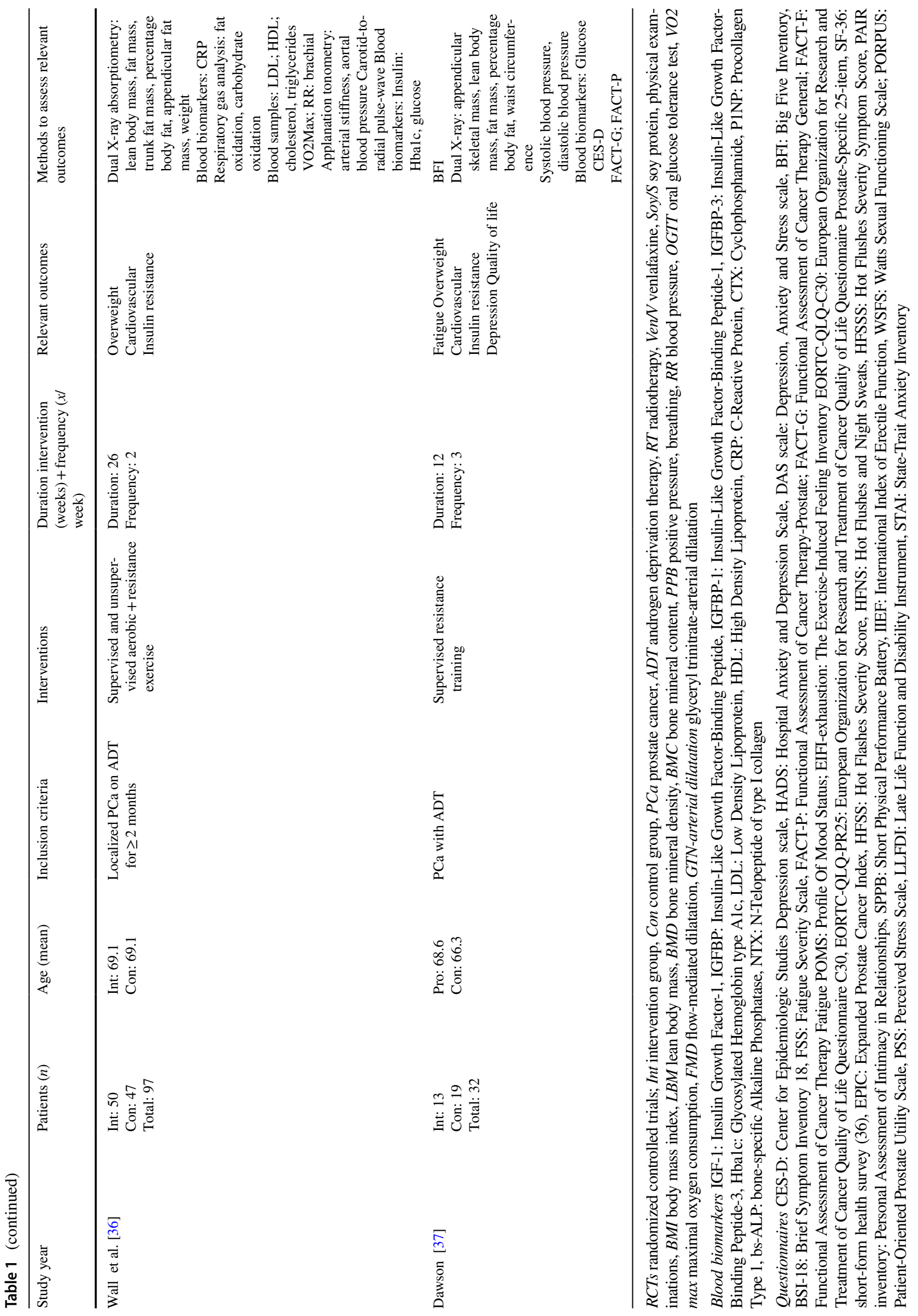


Decreased libido and sexual function Since androgens play an essential role in maintaining sexuality, e.g., libido and erectile function, it is obvious that ADT causes a decrease in sexual activity and may result in a variety of sexual problems. Five studies investigated methods to counteract decreased libido and erectile function [11, 12, $15,19,24]$. Aerobic and resistance exercises were examined in three studies in which contradictory findings were reported. In 2013, Cormie et al. found maintenance of major interest in sex as well as sexual activity in the exercise group. No change in sexual function was noted. In 2015, Cormie et al. reported a diminished decline in sexual function in favor of the exercise group. In per-group analysis, a decline in sexual activity was found without change in sexual function [24]. Culos-Reed et al. found no differences [12].

Patient information concerning the side effects of ADT followed by an educational partner session failed to show an effect on libido, measured by the PAIR and DAS-scores which, respectively, assess the current level of intimacy in one's relationship and the relationship adjustment. Couples participating in the intervention were more successful at maintaining sexual activity [19]. Soy protein consumption did not influence libido or sexual function [11].

\section{Physical side effects}

Gynecomastia Gynecomastia may develop in ADT. In one study, gynecomastia was measured by skinfold thickness and was not influenced by aerobic or resistance training [17].

Hot flushes Hot flushes are defined as intense heat sensation, flushing, and perspiration involving face and trunk. Anxiety and palpitations may occur. ADT may induce these complaints because the decline in LH and FSH causes the release of hypothalamic catecholamines disrupting the thermoregulation center in the upper hypothalamus. CBT temporarily lowers the occurrence of hot flushes and problem rating in men [27]. Only at 6 weeks, significance was reached, and it was no longer significant at 32 weeks. Two studies examined the effect of soy consumption on hot flushes. One study found a significant decrease of ADTassociated vasomotor symptoms in the soy protein and placebo group [18]. Sharma et al. observed no change.

Surprisingly, a significant difference in favor of the placebo group was found [11].

Overweight ADT may cause metabolic effects including dyslipidemia, elevated fasting serum glucose, weight gain, and increase in fat mass. C-reactive protein (CRP) might be a marker of adverse metabolic effects. Seventeen studies reporting on exercise programs of varying duration, frequency, intensity, and degree of supervision showed conflicting results $[9,10,12-14,16,17,20-26,28-32,36$, 37]. These articles mention aspects of body composition amounting to sixteen different items: weight, BMI, waist circumference, hip circumference, waist and neck girth, waist-to-hip ratio, mid-upper arm muscle area, total fat mass, percentage body fat, trunk fat mass, visceral fat mass, body lean mass, appendicular lean mass, and skeletal muscle mass. The effect of aerobic and/or resistance training on body composition was examined in thirteen studies. Three of these studies added dietary advice [14, 20, 28].

Beneficial effects on one or multiple items reflecting body composition were found in eight of these studies (Table 2).

O'neill et al. investigated the effect of aerobic training and dietary advice and showed an improvement in body composition [26]. Two studies examined the effect of supervised football training [21, 32]. One study lasted 12 weeks, the other 32 weeks. Initially, a significant increase in lean body mass was reached after 12 weeks [21]. At 32 weeks, this effect ceased to be significant. Endurance training showed an improvement of body composition [16]. Metabolic syndrome is a clustering of at least three out of five following medical conditions: hypertension, hyperglycemia, abdominal obesity, high serum triglycerides, and a low high-density lipoproteins (HDL).

Blood biomarkers reflecting these changes were examined in six studies. A decrease in HDL/total cholesterol ratio was found by Cormie et al., who investigated the effects of a 12-week program combining aerobic and resistance training [24]. An improvement in HDL was found over time after a program of endurance training [16].

No other studies reported beneficial effects. The influence of aerobic and resistance training on CRP showed contradictory results. One study found a positive effect [13], and two studies found no change [24, 36]. On microscopic level, the effect of resistance training in biopsies of the $\mathrm{m}$. vastus lateralis was reported [29, 30]. An increase in total muscle fibers and type II fibers was shown [29]. Muscle atrophy is associated with reduced mitochondrial function and increased muscle cellular stress, reflected by different heat shock proteins. Only HSP70 improved significantly [30].

Cardiovascular health Factors influencing cardiovascular health are manifold: lipid profile, blood pressure, BMI, endothelial cell function, pro-inflammatory factors, and insulin resistance. ADT has a negative impact on these factors. Besides, there is an association between ADT and the occurrence of serious cardiac arrhythmias. Eleven studies investigated the effect of physical exercise of which three studies added dietary advice $[12,13,16-18,24,26,28,36$, 37]. The results of these studies are contradictory. With respect to blood pressure, four studies found no effect on systolic or diastolic blood pressure between the groups [12, 


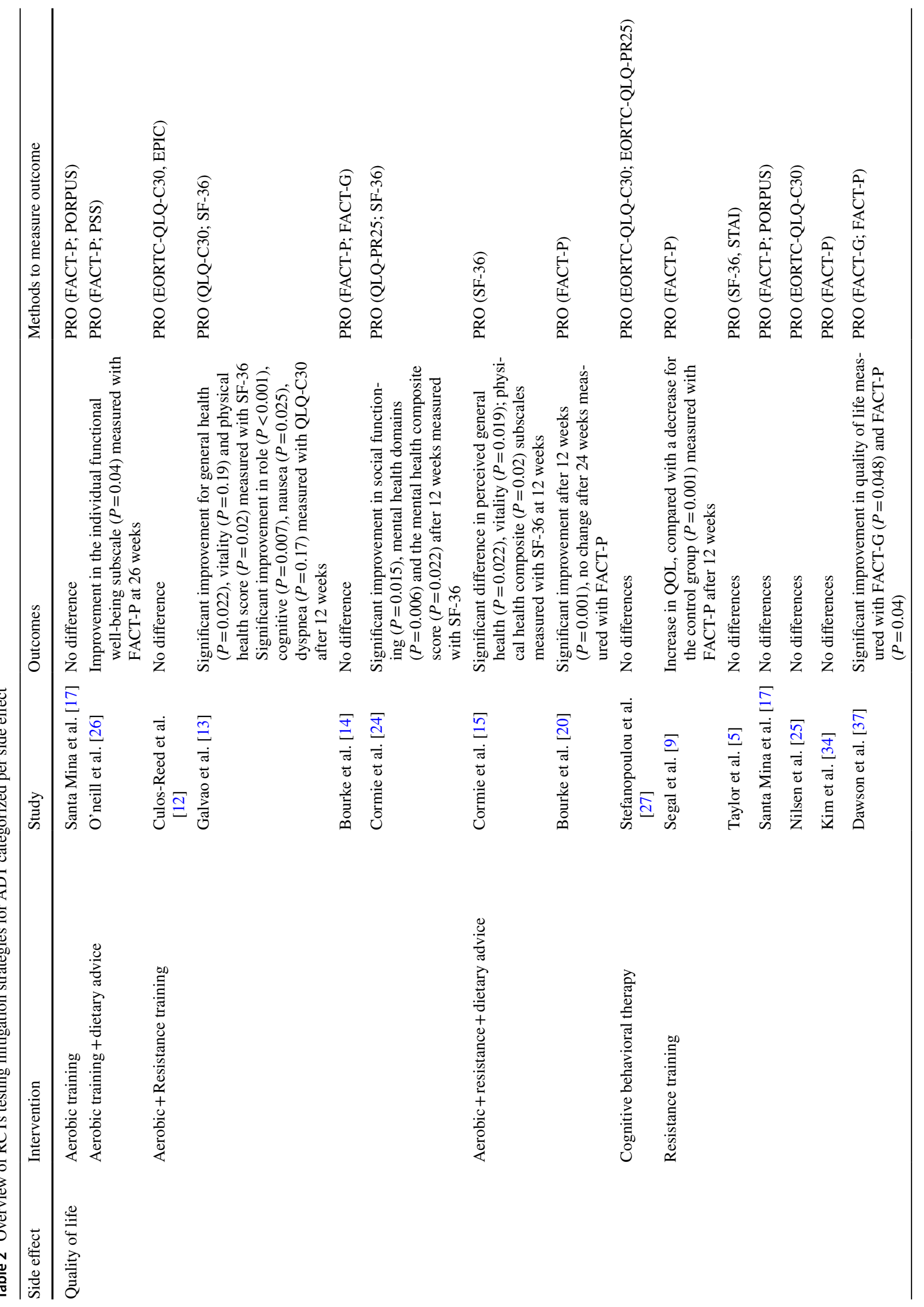




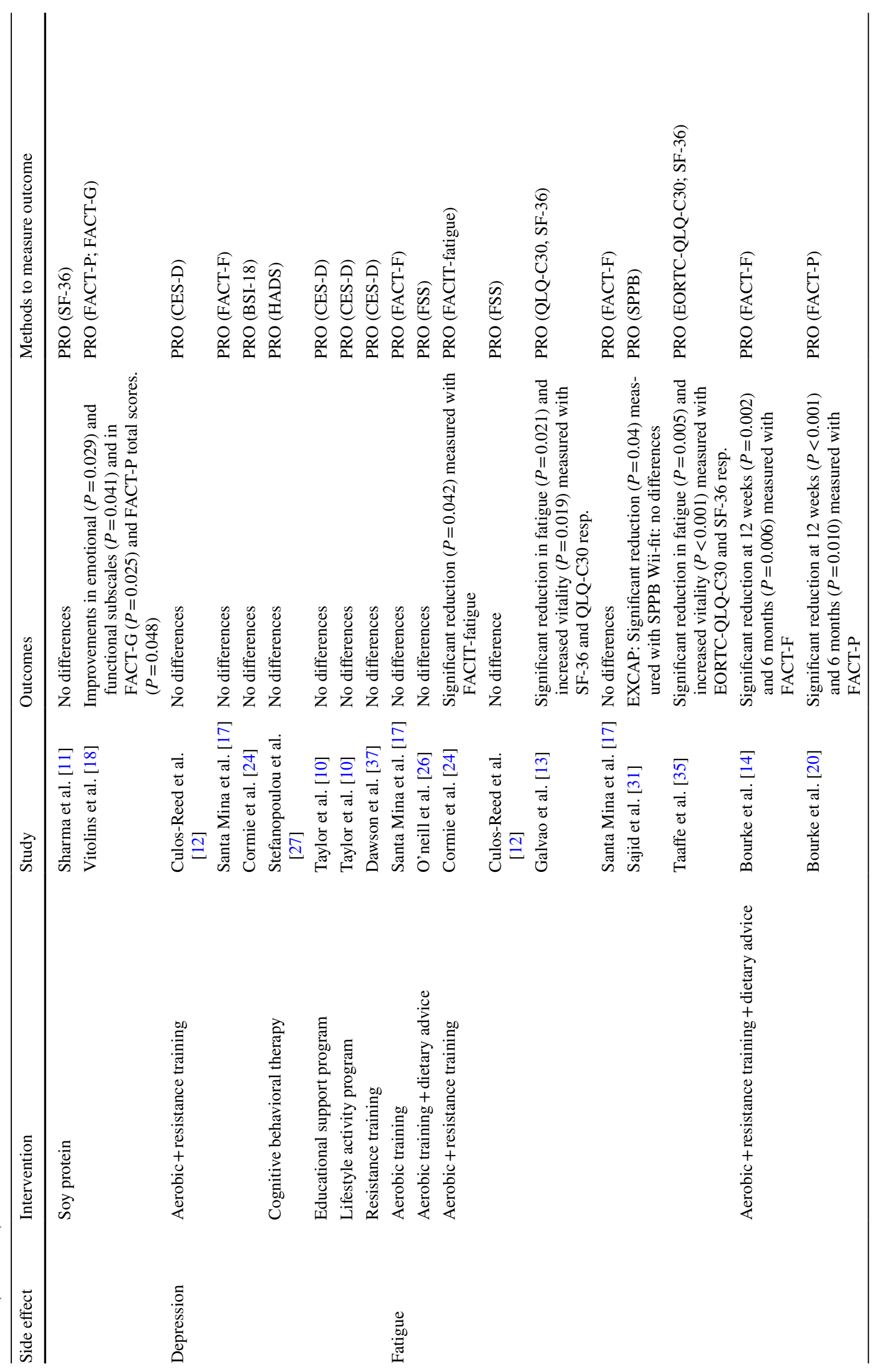




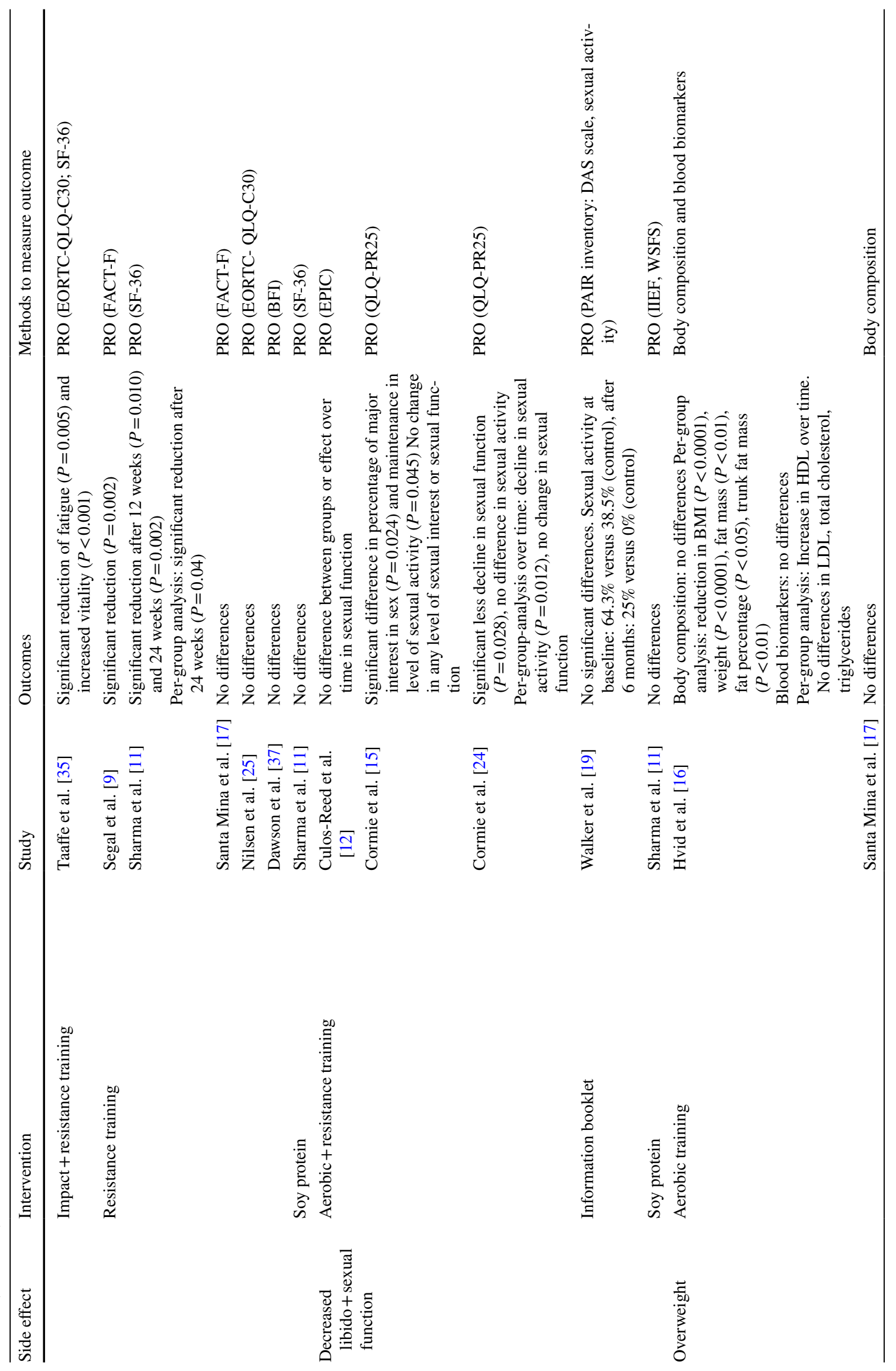




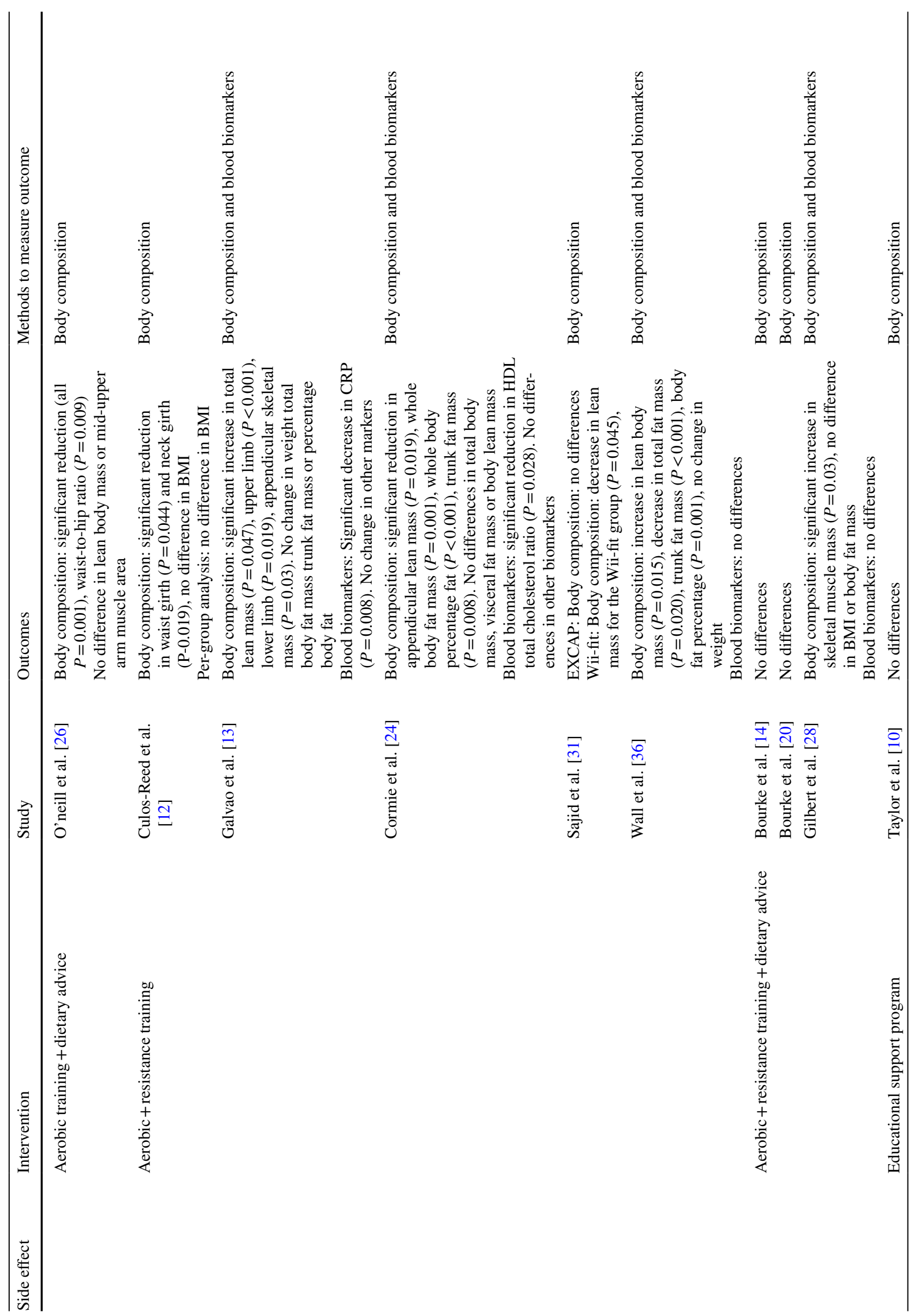




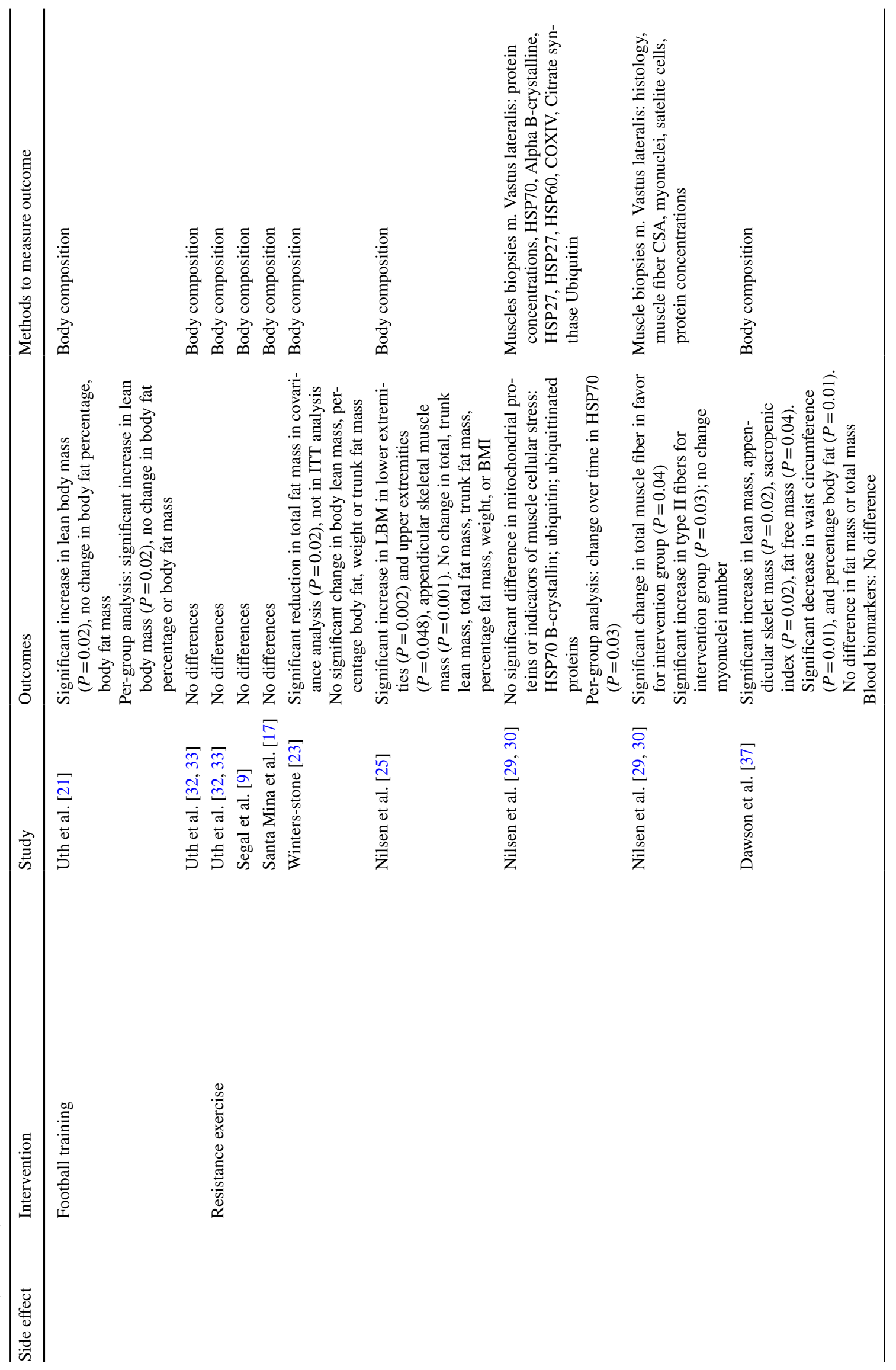




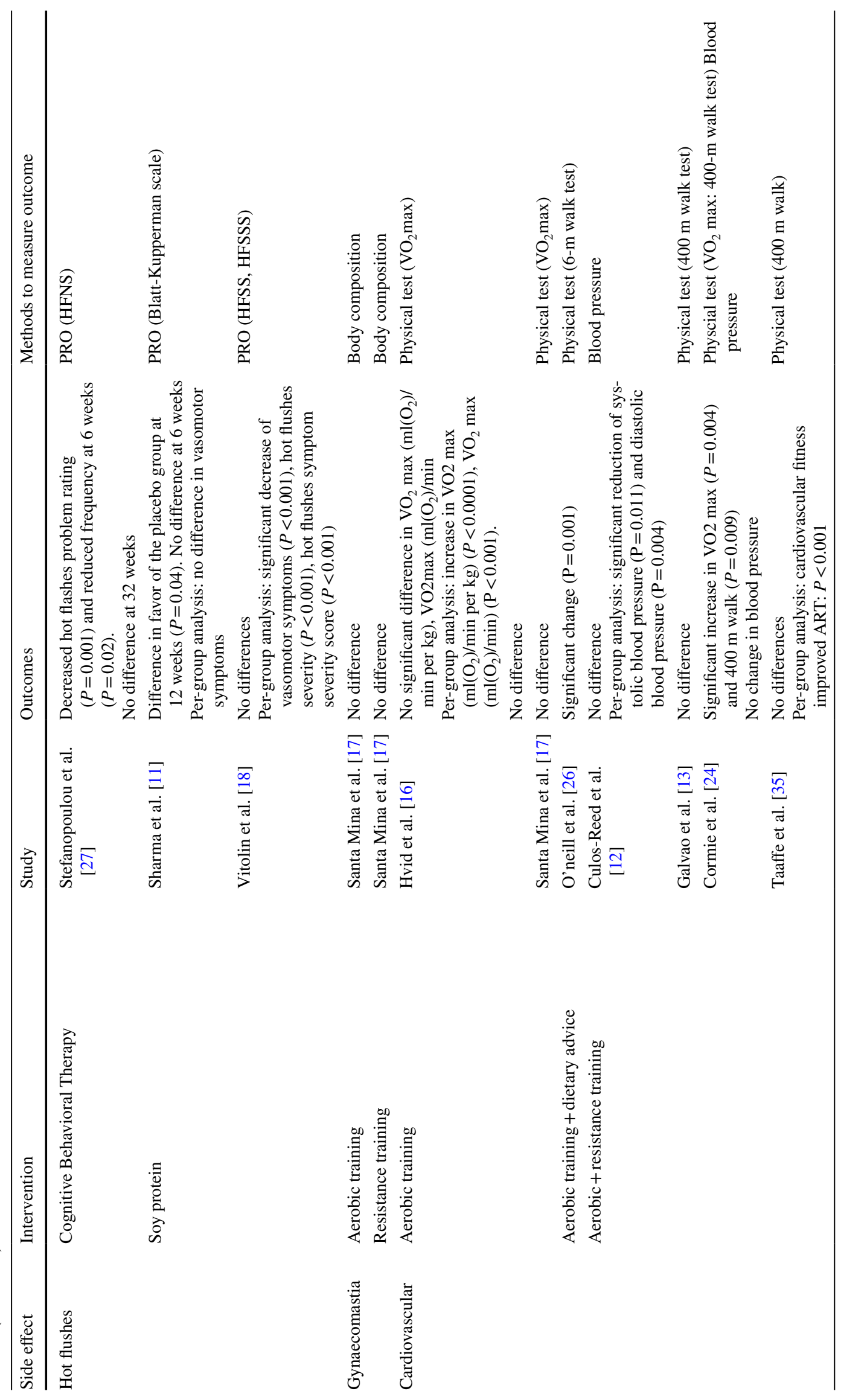




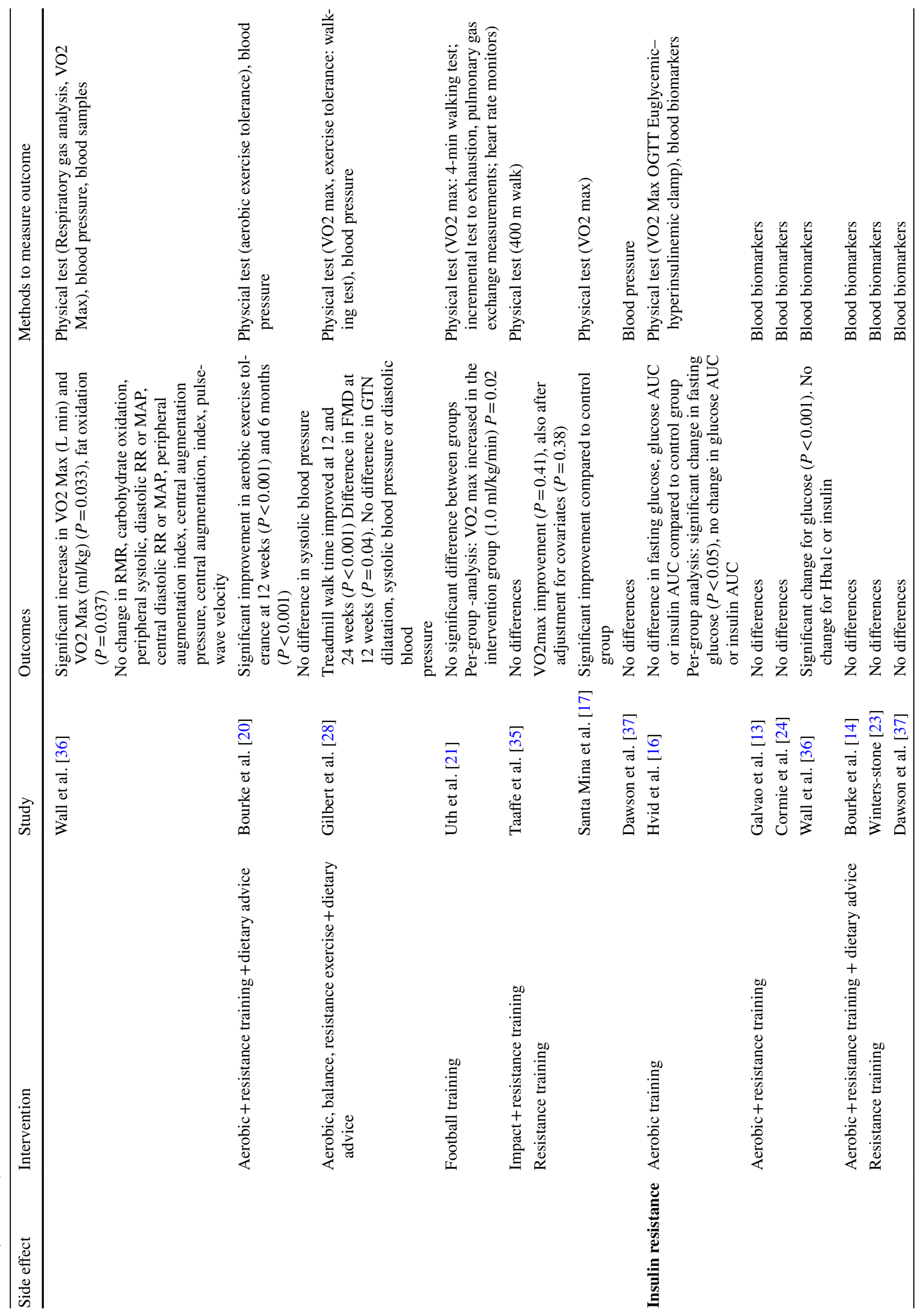




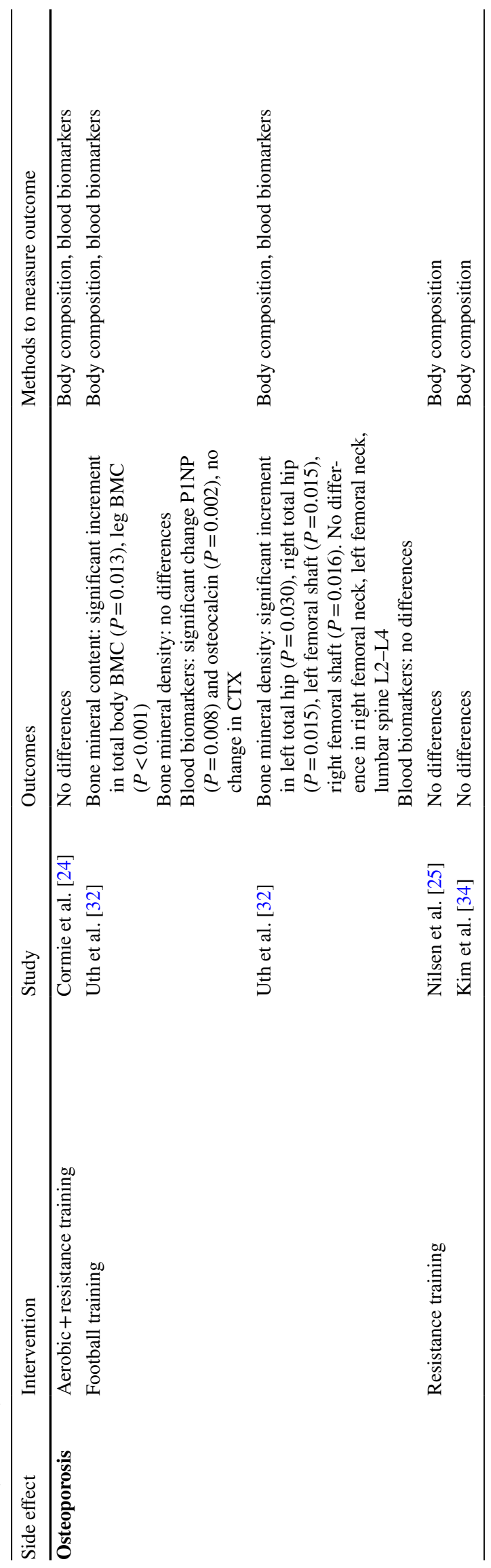


Table 3 Methodological quality assessment tool for randomized controlled trials

\begin{tabular}{|c|c|c|c|c|c|c|c|c|}
\hline & & $\begin{array}{l}\text { Random } \\
\text { sequence gen- } \\
\text { eration (Selec- } \\
\text { tion bias) }\end{array}$ & $\begin{array}{l}\text { Allocation } \\
\text { concealment } \\
\text { (Selection bias) }\end{array}$ & $\begin{array}{l}\text { Blinding of } \\
\text { participants } \\
\text { and personnel } \\
\text { (performance } \\
\text { bias) }\end{array}$ & $\begin{array}{l}\text { Blinding of } \\
\text { outcome assess- } \\
\text { ment (attrition } \\
\text { bias) }\end{array}$ & $\begin{array}{l}\text { Incomplete } \\
\text { outcome data } \\
\text { (attrition bias) }\end{array}$ & $\begin{array}{l}\text { Selective } \\
\text { reporting } \\
\text { (reporting bias) }\end{array}$ & Other bias \\
\hline Segal [9] & 2003 & + & + & - & $?$ & - & + & + \\
\hline Taylor [10] & 2006 & - & - & - & + & + & + & - \\
\hline Sharma [11] & 2009 & + & + & + & + & $?$ & + & - \\
\hline $\begin{array}{l}\text { Culos-Reed } \\
\text { [12] }\end{array}$ & 2010 & + & + & - & + & $?$ & + & - \\
\hline Galvao [13] & 2010 & + & + & - & - & + & + & + \\
\hline Bourke [14] & 2011 & + & $?$ & - & + & + & $?$ & $?$ \\
\hline Cormie [15] & 2013 & + & + & - & $?$ & + & + & + \\
\hline Hvid [16] & 2013 & - & - & - & + & - & + & - \\
\hline Santa Mina [17] & 2013 & $?$ & + & - & + & + & + & - \\
\hline Vitolins [18] & 2013 & + & + & + & + & - & - & - \\
\hline Walker [19] & 2013 & $?$ & $?$ & - & $?$ & + & + & - \\
\hline Bourke [20] & 2014 & + & + & - & - & + & + & + \\
\hline Uth [21] & 2014 & + & + & - & + & + & + & - \\
\hline $\begin{array}{l}\text { Winters-Stone } \\
{[22,23]}\end{array}$ & 2015 & $?$ & $?$ & - & + & + & + & - \\
\hline Cormie [24] & 2015 & + & + & - & $?$ & + & + & + \\
\hline Nilsen [25] & 2015 & + & $?$ & - & + & + & + & + \\
\hline O’Neill [26] & 2015 & + & + & - & - & + & + & $?$ \\
\hline $\begin{array}{l}\text { Stefanopoulou } \\
\text { [27] }\end{array}$ & 2015 & + & + & - & + & - & + & - \\
\hline Gilbert [28] & 2016 & + & + & - & + & + & + & + \\
\hline Nilsen [29] & 2016 & + & $?$ & - & + & - & $?$ & - \\
\hline Nilsen [30] & 2016 & + & $?$ & - & + & - & $?$ & - \\
\hline Sajid [31] & 2016 & + & + & - & + & - & + & + \\
\hline Uth [32] & 2016 & + & + & - & + & + & $?$ & - \\
\hline Uth [33] & 2016 & + & + & - & + & - & + & - \\
\hline Kim [34] & 2017 & + & + & - & - & - & + & - \\
\hline Taaffe [35] & 2017 & + & + & - & + & - & + & $?$ \\
\hline $\begin{array}{c}\text { Wall Dawson } \\
{[36,37]}\end{array}$ & $\begin{array}{l}2017 \\
2018\end{array}$ & $\begin{array}{l}+ \\
+\end{array}$ & $\begin{array}{l}+ \\
+\end{array}$ & - & $\begin{array}{l}+ \\
-\end{array}$ & $\begin{array}{l}- \\
+\end{array}$ & $\begin{array}{l}+ \\
?\end{array}$ & $\begin{array}{l}+ \\
+\end{array}$ \\
\hline
\end{tabular}

Risk of bias summary for each trial included in the systematic review as evaluated by authors

+ low risk of bias; ? Some concerns are risk of bias; - high risk of bias

20, 24, 28]. Culos-Reed et al. found a change in systolic and diastolic blood pressure over time in the aerobic and resistance training group [12]. Using flow-mediated dilatation (FMD) as a measure, an improvement in the exercise combined with dietary advice group was found [28]. The effect of training on maximum oxygen utilization (VO2 max) was investigated in five studies [17, 18, 24, 36, 37]. Most of these studies consisted of a mixture of aerobic and/or resistance training. Uth et al. used football training. Almost consistently, an improvement was found.

Resistance training scored better in increasing cardiovascular fitness than aerobic $[17,18]$. Four studies found a significant improvement on cardiovascular fitness after physical exercises, measured by different tests [13, 26, 29, 35].

Insulin resistance It is not completely understood how ADT therapy deregulates glucose metabolism. Possibly hypogonadism, secondary to obesity, alters fatty acid metabolism or changes in skeletal muscle may play a role. The influence of exercise on insulin resistance was measured in seven studies [13, 14, 16, 22-24, 36, 37]. One study added dietary advice. A glucose-lowering effect of exercise was shown in two studies [16, 36]. Wall et al. showed an improvement for glucose metabolism in combined aerobic and resistance training [36]. Hvid et al. investigated the effect of endurance 
training on insulin resistance in men on ADT compared to healthy males and found a difference in fasting glucose over time in the per-group analysis [16].

Using the oral glucose tolerance test (OGTT), no differences over time were found between the groups with regard to fasting glucose, fasting insulin, glucose AUC, and insulin AUC [16]. Biomarkers: IGF-1, insulin, IFGBP-1 and IGFBP-3, and HBA1c failed to show a difference in any of the studies.

Osteoporosis Osteoporosis is a major concern in men undergoing ADT therapy, especially with prolonged use. Five studies investigated the effect of exercise modalities on osteoporosis [24, 25, 32-34]. We were unable to find any studies focusing on dietary advice. Dual x-ray absorptiometry (DEXA-scan) was used in all studies in order to examine bone mineral density (BMD) and bone mineral content (BMC). Blood biomarkers were examined in four studies. Biomarkers were alkaline phosphatase, P1NP, N-telopeptide, N-telopeptide/creatinine ratio, vitamin $\mathrm{D}$, osteocalcin, CTX, NTX, and BS-ALP. Only Uth was able to show a significant improvement in different markers of osteoporosis. He found an increment in BMC after 12 weeks of supervised football training [33]. Initially, BMD remained unchanged after 12 weeks of training [33], whereas after 32 weeks, an increase in BMD was found in the total hip and femoral shaft [32]. P1NP and osteocalcin were the only bone formation markers that showed a significant change. After 12 weeks, there was an increase in these markers [32] After prolonged training (32 weeks), this increase no longer was significant [32].

\section{Discussion}

This study focuses on lifestyle interventions to reduce the deleterious side effects of ADT. Twenty-nine RCTs reported the effect of different lifestyle interventions. The effect of exercises in different modalities and intensities was studied including measures such as dietary advice, self-education, CBT, or educational support program. We excluded therapies like prescribing calcium and vitamin D.

Regarding psychological effects, contradictory results were found. Many different questionnaires were used to examine the effect of lifestyle interventions on HRQOL, which make it hard to compare different outcomes and therefore it is difficult to make a straightforward conclusion about the effect of certain interventions.

Combining exercise with diet seems to have the most beneficial effects on HRQOL; all studies regarding this combination showed a significant improvement $[15,20,26]$. Soy protein consumption was found in only one study to have a beneficial effect.
An earlier study found that ADT use conferred a $41 \%$ increased risk of depression [38]. In this review, we were unable to find any difference in the occurrence of depression after applying lifestyle interventions, whereas in two studies an improvement of QOL was noted in absence of improvement of depression score [24, 37]. Tentative explanations for these outcomes can be the following. Both studies used different questionnaires to score quality of life and the occurrence of depression, making it difficult to compare them. In our review, we only noted the truly significant findings, but in the study of Cormie, where an improvement in various subscales of QOL was observed, a borderline significance was found in the depression score with a $P$ value of 0054 ; this might be interpreted as a trend in improvement of physiological well-being [24].

There were many different tools used to examine fatigue. Some used general questionnaires, and others applied prostate-specific tools (FACT-F) [9, 14, 17, $20,24]$. Although there was a lack in using a common tool, different lifestyle interventions reduced fatigue and increased vitality. All studies used in this review examined physical exercises, sometimes combined with dietary advice. However, no attention was given to other lifestyle adjustments, such as smoking, alcohol use, or a balanced diet.

With regard to libido and sexual function, a possible beneficial effect of aerobic and resistance training was found $[15,24]$. It is noteworthy that consuming soy protein did not have any effect [11]. Considering the age group for which ADT therapy is applied, we can expect a general natural decline of libido and sexual function.

Gynecomastia may very well occur after weight gain. Only one author reported on this subject [17]. This might reflect a disbelief in the effect of lifestyle changes on gynecomastia. A variety of medical therapies exist such as radiation therapy or lipolysis [39].

Two strategies were examined to reduce vasomotor symptoms. Although soy proteins appears to be successful in treating hot flushes in postmenopausal women [40], in men on ADT, they are unsuccessful. CBT appears to be helpful in managing hot flushes. Limitations of this study were the small sample size and short period of self-guided therapy. Further research might look into additional variables such as caffeine intake and other specific health problems.

In this study, we divided the metabolic changes into three subcategories: overweight, insulin resistance, and cardiovascular health. These categories are interrelated. Concerning insulin resistance, studies failed to show a difference between men with ADT undergoing physical exercises or not, when measured by fasting glucose. Endurance training, however, showed no differences in OGTT between men on ADT undergoing exercises versus healthy men [16]. No trial used the novo diabetes mellitus as an outcome. 
Overweight was examined in 17 studies. All studies investigated the effect of exercise, and four studies added nutritional advice. Evidence was found that solely exercise or exercise combined with nutritional advice may decrease overweight. The influence of a dietary advice only on obesity hasn't been investigated. Earlier studies showed the major impact of an appropriate diet in the prevention of obesity in the healthy old male population [41].

Considering osteoporosis, one study suggests that regularly practicing football may mitigate ADT-induced decline in BMD. However, aerobic or resistance training failed to show this decline. Future studies should investigate the effects of different exercise modalities.

National osteoporosis foundation recommends a daily calcium intake of $1200 \mathrm{mg}$ and supplemental vitamin D of 800-1000 IU to maintain bone health [42]. In this review, there was not one study, which focused on an adequate intake as described above. As mentioned earlier, we did not include RCTs in which calcium and Vitamin D were prescribed. A systematic review conducted in 2012, which investigated the effect of calcium and vitamin D suppletion in men with PCa using ADT showed conflicting effects [43]. It would be interesting to conduct studies in which dietary advice is given to promote sufficient intake of calcium and vitamin D and investigate this effect on the development of osteoporosis in patients on ADT.

A number of limitations in this review must be considered. Firstly, we focused on two databases, PubMed and Medline, since these two are the most widely used and recognized. Secondly, there was substantial heterogeneity in life style interventions, in which some interventions were investigated frequently, while other interventions only once. This makes it impossible to compare the effects of different strategies and draw conclusions about the efficacy of these interventions. Additionally, we did not investigate the effect of duration of exercise, dietary advice, or other behavioral components.

There was a variety in quality of the different RCTs. Especially, the prevention of performance bias was often inadequate, due to the fact it was impossible to blind participants following lifestyle programs. Despite these limitations, evidence suggests that lifestyle interventions may have a beneficial effect on ADT-mediated side effects.

\section{Conclusions}

Adverse effects of ADT are manifold as well as the various lifestyle interventions aimed to alleviate these side effects. Physical exercises demonstrated to have a mainly positive effect on cardiovascular health. Contradictory results were found regarding quality of life, libido, fatigue, insulin resistance, overweight, and osteoporosis. No effect was found regarding depression and gynecomastia. Regarding dietary advice, soy protein showed no beneficial effect on libido or fatigue. No other studies allowed conclusions on dietary advice solely. A positive effect was shown with CBT on the occurrence of hot flushes. Other behavioral components failed to show a significant effect regarding side effects. Further research is necessary to identify the most effective interventions for the individual patient.

Funding This study was funded by AstraZeneca BV, the Netherlands.

\section{Compliance with ethical standards}

Conflict of interest The authors declare that they have no conflict of interest.

Open Access This article is distributed under the terms of the Creative Commons Attribution 4.0 International License (http://creativeco mmons.org/licenses/by/4.0/), which permits unrestricted use, distribution, and reproduction in any medium, provided you give appropriate credit to the original author(s) and the source, provide a link to the Creative Commons license, and indicate if changes were made.

\section{References}

1. Siegel, R. L., Miller, K. D., \& Jemal, A. (2018). Cancer statistics, 2018. CA: A Cancer Journal for Clinicians, 68(1), 7-30. https:// doi.org/10.3322/caac.21442.

2. Huggins, C. (1941). Studies on prostatic cancer. I. The effect of castration, of estrogen and of androgen injection on serum phosphatases in metastatic carcinoma of the prostate. Cancer Research, 1(4), 293.

3. Heidenreich, A., Bastian, P. J., Bellmunt, J., et al. (2014). EAU guidelines on prostate cancer. Part II: Treatment of advanced, relapsing, and castration-resistant prostate cancer. European Urology, 65(2), 467-479. https://doi.org/10.1016/j.eurur o.2013.11.002.

4. Denis, L., \& Murphy, G. P. (1993). Overview of phase III trials on combined androgen treatment in patients with metastatic prostate cancer. Cancer, 72(12 Suppl), 3888-3895.

5. Taylor, L. G., Canfield, S. E., \& Du, X. L. (2009). Review of major adverse effects of androgen-deprivation therapy in men with prostate cancer. Cancer, 115(11), 2388-2399. https://doi.org/10.1002/ cncr.24283.

6. Yunfeng, G., Weiyang, H., Xueyang, H., Yilong, H., \& Xin, G. (2017). Exercise overcome adverse effects among prostate cancer patients receiving androgen deprivation therapy: An update metaanalysis. Medicine (Baltimore)., 96(27), e7368.

7. Gardner, Jason R., Livingston, Patricia M., \& Fraser, Steve F. (2014). Effects of exercise on treatment-related adverse effects for patients with prostate cancer receiving androgen-deprivation therapy: a systematic review. Journal of Clinical Oncology, 32(4), 335-346.

8. Higgins, J. P. T., \& Green, S. (2011). Cochrane handbook for systematic reviews of interventions (Vol. 4). Hoboken: Wiley.

9. Segal, R. J., Reid, R. D., Courneya, K. S., et al. (2003). Resistance exercise in men receiving androgen deprivation therapy for prostate cancer. Journal of Clinical Oncology, 21(9), 1653-1659. https://doi.org/10.1200/JCO.2003.09.534. 
10. Carmack Taylor, C. L., Demoor, C., Smith, M. A., et al. (2006). Active for Life After Cancer: A randomized trial examining a lifestyle physical activity program for prostate cancer patients. Psychooncology., 15(10), 847-862. https://doi.org/10.1002/pon.1023.

11. Sharma, P., Wisniewski, A., Braga-Basaria, M., et al. (2009). Lack of an effect of high dose isoflavones in men with prostate cancer undergoing androgen deprivation therapy. Journal of Urology, 182(5), 2265-2272. https://doi.org/10.1016/j.juro.2009.07.030.

12. Culos-Reed, S. N., Robinson, J. W., Lau, H., et al. (2010). Physical activity for men receiving androgen deprivation therapy for prostate cancer: Benefits from a 16-week intervention. Support Care Cancer, 18(5), 591-599. https://doi.org/10.1007/s0052 0-009-0694-3.

13. Galvao, D. A., Taaffe, D. R., Spry, N., Joseph, D., \& Newton, R. U. (2010). Combined resistance and aerobic exercise program reverses muscle loss in men undergoing androgen suppression therapy for prostate cancer without bone metastases: A randomized controlled trial. Journal of Clinical Oncology, 28(2), 340-347. https://doi.org/10.1200/JCO.2009.23.2488.

14. Bourke, L., Doll, H., Crank, H., Daley, A., Rosario, D., \& Saxton, J. M. (2011). Lifestyle intervention in men with advanced prostate cancer receiving androgen suppression therapy: A feasibility study. Cancer Epidemiology, Biomarkers \& Prevention, 20(4), 647-657. https://doi.org/10.1158/1055-965.EPI-10-1143.

15. Cormie, P., Newton, R. U., Taaffe, D. R., et al. (2013). Exercise maintains sexual activity in men undergoing androgen suppression for prostate cancer: A randomized controlled trial. Prostate Cancer Prostatic Dis., 16(2), 170-175. https://doi.org/10.1038/ pcan.2012.52.

16. Hvid, T., Winding, K., Rinnov, A., et al. (2013). Endurance training improves insulin sensitivity and body composition in prostate cancer patients treated with androgen deprivation therapy. Endocrine-Related Cancer, 20(5), 621-632. https://doi.org/10.1530/ ERC-12-0393.

17. Santa Mina, D., Alibhai, S. M. H., Matthew, A. G., et al. (2013). A randomized trial of aerobic versus resistance exercise in prostate cancer survivors. Journal of Aging and Physical Activity, 21(4), 455-478.

18. Vitolins, M. Z., Griffin, L., Tomlinson, W. V., et al. (2013). Randomized trial to assess the impact of venlafaxine and soy protein on hot flashes and quality of life in men with prostate cancer. Journal of Clinical Oncology, 31(32), 4092-4098. https://doi.org/10.1200/ JCO.2012.48.1432.

19. Walker, L. M., Hampton, A. J., Wassersug, R. J., Thomas, B. C., \& Robinson, J. W. (2013). Androgen deprivation therapy and maintenance of intimacy: A randomized controlled pilot study of an educational intervention for patients and their partners. Contemp Clin Trials., 34(2), 227-231. https://doi.org/10.1016/j. cct.2012.11.007.

20. Bourke, L., Gilbert, S., Hooper, R., et al. (2014). Lifestyle changes for improving disease-specific quality of life in sedentary men on long-term androgen-deprivation therapy for advanced prostate cancer: A randomised controlled trial. European Urology, 65(5), 865-872. https://doi.org/10.1016/j.eururo.2013.09.040.

21. Uth, J., Hornstrup, T., Schmidt, J. F., et al. (2014). Football training improves lean body mass in men with prostate cancer undergoing androgen deprivation therapy. Scandinavian Journal of Medicine and Science in Sports, 24(Suppl 1), 105-112. https:// doi.org/10.1111/sms.12260.

22. Winters-Stone, K. M., Dieckmann, N., Maddalozzo, G. F., Bennett, J. A., Ryan, C. W., \& Beer, T. M. (2015). Resistance exercise reduces body fat and insulin during androgen-deprivation therapy for prostate cancer. Oncology Nursing Forum, 42(4), 348-356. https://doi.org/10.1188/15.ONF.348-356.

23. Winters-Stone, K. M., Dobek, J. C., Benett, J. A., et al. (2015). Resistance training reduces disability in prostate cancer survivors on androgen deprivation therapy: Evidence from a randomized controlled trial. Archives of Physical Medicine and Rehabilitation, 96(1), 7-14. https://doi.org/10.1016/j.apmr.2014.08.010.

24. Cormie, P., Galvao, D. A., Spry, N., et al. (2015). Can supervised exercise prevent treatment toxicity in patients with prostate cancer initiating androgen-deprivation therapy: A randomised controlled trial. BJU International, 115(2), 256-266. https://doi.org/10.1111/ bju. 12646.

25. Nilsen, T. S., Raastad, T., Skovlund, E., et al. (2015). Effects of strength training on body composition, physical functioning, and quality of life in prostate cancer patients during androgen deprivation therapy. Acta Oncologica, 54(10), 1805-1813. https://doi. org/10.3109/0284186X.2015.1037008.

26. O’Neill, R. F., Haseen, F., Murray, L. J., O’Sullivan, J. M., \& Cantwell, M. M. (2015). A randomised controlled trial to evaluate the efficacy of a 6-month dietary and physical activity intervention for patients receiving androgen deprivation therapy for prostate cancer. Journal of Cancer Survivorship, 9(3), 431-440. https:// doi.org/10.1007/s11764-014-0417-8.

27. Stefanopoulou, E., Yousaf, O., Grunfeld, E. A., \& Hunter, M. S. (2015). A randomised controlled trial of a brief cognitive behavioural intervention for men who have hot flushes following prostate cancer treatment (MANCAN). Psychooncology, 24(9), 1159-1166. https://doi.org/10.1002/pon.3794.

28. Gilbert, S. E., Tew, G. A., Fairhurst, C., et al. (2016). Effects of a lifestyle intervention on endothelial function in men on long-term androgen deprivation therapy for prostate cancer. British Journal of Cancer, 114(4), 401-408. https://doi.org/10.1038/bjc.2015.479.

29. Nilsen, T. S., Thorsen, L., Kirkegaard, C., Ugelstad, I., Fossa, S. D., \& Raastad, T. (2016). The effect of strength training on muscle cellular stress in prostate cancer patients on ADT. Endocr Connect., 5(2), 74-82. https://doi.org/10.1530/EC-15-0120.

30. Nilsen, T. S., Thorsen, L., Fossa, S. D., et al. (2016). Effects of strength training on muscle cellular outcomes in prostate cancer patients on androgen deprivation therapy. Scandinavian Journal of Medicine and Science in Sports, 26(9), 1026-1035. https://doi. org/10.1111/sms.12543.

31. Sajid, S., Dale, W., Mustian, K., et al. (2016). Novel physical activity interventions for older patients with prostate cancer on hormone therapy: A pilot randomized study. J Geriatr Oncol., 7(2), 71-80. https://doi.org/10.1016/j.jgo.2016.02.002.

32. Uth, J., Hornstrup, T., Christensen, J. F., et al. (2016). Efficacy of recreational football on bone health, body composition, and physical functioning in men with prostate cancer undergoing androgen deprivation therapy: 32-week follow-up of the FC prostate randomised controlled trial. Osteoporosis International, 27(4), 1507-1518. https://doi.org/10.1007/s00198-015-3399-0.

33. Uth, J., Hornstrup, T., Christensen, J. F., et al. (2016). Football training in men with prostate cancer undergoing androgen deprivation therapy: Activity profile and short-term skeletal and postural balance adaptations. European Journal of Applied Physiology, 116(3), 471-480. https://doi.org/10.1007/s00421-015-3301-y.

34. Kim, S. H., Seong, D. H., Yoon, S. M., et al. (2017). The effect on bone outcomes of home-based exercise intervention for prostate cancer survivors receiving androgen deprivation therapy: A pilot randomized controlled trial. Cancer Nursing. https://doi. org/10.1097/NCC.0000000000000530.

35. Taaffe, D. R., Newton, R. U., Spry, N., et al. (2017). Effects of different exercise modalities on fatigue in prostate cancer patients undergoing androgen deprivation therapy: A year-long randomised controlled trial. European Urology, 72(2), 293-299. https://doi.org/10.1016/j.eururo.2017.02.019.

36. Wall, B. A., Galvao, D. A., Fatehee, N., et al. (2017). Exercise Improves $\mathrm{VO}_{2 \max }$ and body composition in androgen deprivation therapy-treated prostate cancer patients. Medicine \& Science in 
Sports \& Exercise, 49(8), 1503-1510. https://doi.org/10.1249/ mss.0000000000001277.

37. Dawson, J. K., Dorff, T. B., Todd Schroeder, E., et al. (2018). Impact of resistance training on body composition and metabolic syndrome variables during androgen deprivation therapy for prostate cancer: A pilot randomized controlled trial. BMC Cancer, 18(1), 368. https://doi.org/10.1186/s12885-018-4306-9.

38. Nead, K. T., Sinha, S., Yang, D. D., \& Nguyen, P. L. (2017). Association of androgen deprivation therapy and depression in the treatment of prostate cancer: A systematic review and metaanalysis. Urologic Oncology, 35(11), 664.e1-664.e9. https://doi. org/10.1016/j.urolonc.2017.07.016.

39. Widmark, A., Fossa, S. D., Lundmo, P., et al. (2003). Does prophylactic breast irradiation prevent antiandrogen-induced gynecomastia? Evaluation of 253 patients in the randomized Scandinavian trial SPCG-7/SFUO-3. Urology, 61(1), 145-151.

40. Husain, D., Khanna, K., Puri, S., \& Haghighizadeh, M. (2015). Supplementation of soy isoflavones improved sex hormones, blood pressure, and postmenopausal symptoms. Journal of the American College of Nutrition, 34(1), 42-48. https://doi. org/10.1080/07315724.2013.875434.
41. Cermak, N. M., Res, P. T., de Groot, L. C., Saris, W. H., \& van Loon, L. J. (2012). Protein supplementation augments the adaptive response of a skeletal muscle to resistance-type exercise training: A meta-analysis. The American Journal of Clinical Nutrition, 96, 1454-1464.

42. Weaver, C. M., Alexander, D. D., Boushey, C. J., et al. (2016). Calcium plus vitamin D supplementation and risk of fractures: An updated meta-analysis from the National Osteoporosis Foundation. Osteoporosis International, 27(1), 367-376. https://doi. org/10.1007/s00198-015-3386-5.

43. Datta, M., \& Schwartz, G. G. (2012). Calcium and vitamin d supplementation during androgen deprivation therapy for prostate cancer: A Critical review. Oncologist., 17(9), 1171-1179. https ://doi.org/10.1634/theoncologist.2012-0051.

Publisher's Note Springer Nature remains neutral with regard to jurisdictional claims in published maps and institutional affiliations. 